\title{
ON STABILIZERS OF SOME TEICHMÜLLER DISKS IN POINTED MAPPING CLASS GROUPS
}

\author{
C. ZHANG \\ Department of Mathematical Sciences, Morehouse College, Atlanta, GA 30314, USA \\ e-mail: czhang@morehouse.edu
}

(Received 28 June 2009; accepted 7 April 2010)

\begin{abstract}
We prove that for each Riemann surface $\tilde{S}$ of finite analytic type $(p, n)$ with $p \geq 2$, there exist uncountably many Teichmüller disks $\Delta$ in the Teichmüller space $T(S)$, where $S=\tilde{S}-$ a point $a$ \}, with these properties: (1) the natural projection $j: T(S) \rightarrow T(\tilde{S})$ defined by forgetting $a$ induces an isometric embedding of each $\Delta$ into $T(\tilde{S})$; and (2) the stabilizer of each Teichmüller disk $\Delta$ in the $a$-pointed mapping class group of $S$ is trivial.
\end{abstract}

2010 Mathematics Subject Classification. 30C60, 30F60.

1. Introduction. Let $\tilde{S}$ be a Riemann surface of type $(p, n)$, where $p$ is the genus and $n$ is the number of punctures. Assume that $3 p-3+n>0$. Let $\operatorname{Homeo}(\tilde{S})$ be the group of orientation preserving self-homeomorphisms of $\tilde{S}$ and let $\operatorname{Homeo}_{0}(\tilde{S})$ be the subgroup consisting of those maps isotopic to the identity. The Teichmüller space $T(\tilde{S})$ is the space of all conformal structures on $\tilde{S}$ quotient by $\operatorname{Homeo}_{0}(\tilde{S})$. The mapping class $\operatorname{group}_{\operatorname{Mod}_{\tilde{S}}}$ defined by the quotient $\operatorname{Homeo}(\tilde{S}) / \operatorname{Homeo}_{0}(\tilde{S})$ acts on $T(\tilde{S})$ as a group of holomorphic automorphisms; and also as a group of isometries if $T(\tilde{S})$ is endowed with the standard Teichmüller distance $d(\cdot, \cdot)$. See Abikoff [1] for more details.

There is a smooth fibre bundle $V(\tilde{S}) \rightarrow T(\tilde{S})$ whose fibre $V_{x}$ over a point $x \in T(\tilde{S})$ is a Riemann surface representing $x$. The Bers fibre space $\pi: F(\tilde{S}) \rightarrow T(\tilde{S})$ is defined as the composition

$$
F(\tilde{S}) \rightarrow V(\tilde{S}) \rightarrow T(\tilde{S})
$$

where $F(\tilde{S}) \rightarrow V(\tilde{S})$ is the universal covering map. The central fibre of $F(\tilde{S})$ is the hyperbolic plane $\mathbf{H}=\{z \in \mathbf{C}: \operatorname{Im} z>0\}$. Let $G$ be the covering group of the universal covering map $\varrho: \mathbf{H} \rightarrow \tilde{S}$. Then $G$ is a Fuchsian group such that $\mathbf{H} / G=\tilde{S}$. Note that every point $x \in T(\tilde{S})$ is represented by a conformal structure $\sigma$ on $\tilde{S}$ that is lifted to a measurable function $\sigma(z)$ on $\mathbf{H}$ with these properties: (i) ess. $\sup \{|\sigma(z)| ; z \in \mathbf{H}\}<1$ and (ii) $\sigma(g(z)) \overline{g^{\prime}(z)} / g^{\prime}(z)=\sigma(z)$ for all $g \in G$ and $z \in \mathbf{H}$. The fibre $\mathscr{F}_{x} \subset F(\tilde{S})$ over a point $x \in T(\tilde{S})$ is the quasi-disk $w^{\sigma}(\mathbf{H})$, where $w^{\sigma}: \hat{\mathbf{C}} \rightarrow \hat{\mathbf{C}}$ is, according to Ahlfors and Bers [2], a quasi-conformal map fixing $0,1, \infty$ and satisfying

$$
\frac{\partial_{\bar{z}} w^{\sigma}(z)}{\partial_{z} w^{\sigma}(z)}= \begin{cases}\sigma(z) & \text { if } z \in \mathbf{H}, \\ 0 & \text { if } z \in \mathbf{C}-\overline{\mathbf{H}} .\end{cases}
$$


Let $\tilde{\theta} \in \operatorname{Mod}_{\tilde{S}}$ be a hyperbolic element; that is, there is a point $x \in T(\tilde{S})$ such that $d(x, \tilde{\theta}(x))>0$ and

$$
\inf d\left(x^{\prime}, \tilde{\theta}\left(x^{\prime}\right)\right)=d(x, \tilde{\theta}(x)),
$$

where the infimum is taken over all $x^{\prime} \in T(\tilde{S})$. This implies that $\tilde{\theta}$ is represented by an absolutely extremal Teichmüller self-map $\tilde{\omega}$ on a surface (also called $\tilde{S}$ ) that determines a holomorphic quadratic differential $\phi_{\tilde{\omega}}$ on $\tilde{S}$, which may have simple poles at punctures of $\tilde{S}$ and satisfies

$$
\iint_{\tilde{S}}\left|\phi_{\tilde{\omega}}(z)\right| d x d y=1 .
$$

Note that all zeros of $\phi_{\tilde{\omega}}$ may be punctures of $\tilde{S}$. Denote by $\mu=\bar{\phi}_{\tilde{\omega}} /\left|\phi_{\tilde{\omega}}\right|$. Then $\mu$ is a $(1,1)$-form on $\tilde{S}$. Let $[v]$ denote the equivalence class of a conformal structure $v$ on $\tilde{S}$. Let $\mathbf{D}=\{t:|t|<1\}$ be the unit disk. We define a Teichmüller disk

$$
\tilde{\Delta}_{\tilde{\omega}}=\{[t \mu]: t \in \mathbf{D}\} \subset T(\tilde{S}) .
$$

By Theorem 5 of Bers [6], $\tilde{\Delta}_{\tilde{\omega}}$ is the unique invariant disk under the action of $\tilde{\theta}$. Associated to each point $\hat{z} \in \mathbf{H}$ there is a disk

$$
\mathscr{D}_{\tilde{\omega}}(\hat{z})=\left\{\left([t \mu], w^{t \mu}(\hat{z})\right): t \in \mathbf{D}\right\} \subset F(\tilde{S}) .
$$

Let $a \in \tilde{S}$ be a point and let $S=\tilde{S}-\{a\}$. The Bers isomorphism theorem [5] states that there is an isomorphism $\varphi: F(\tilde{S}) \rightarrow T(S)$ that is determined up to a modular transformation of $T(S)$ so that

$$
j=\pi \circ \varphi^{-1}: T(S) \rightarrow T(\tilde{S})
$$

is the natural forgetful map. Due to the Bers isomorphism theorem, one proves (Kra [11]) that the embedding

$$
\Delta_{\tilde{\omega}}(\hat{z})=\varphi\left(\mathscr{D}_{\tilde{\omega}}(\hat{z})\right) \subset T(S)
$$

is a Teichmüller disk so that $j\left(\Delta_{\tilde{\omega}}(\hat{z})\right)=\tilde{\Delta}_{\tilde{\omega}}$. Let $\operatorname{Mod}_{S}^{a}$ denote the $a$-pointed mapping class group $\operatorname{Mod}_{S}^{a}$. That is, $\operatorname{Mod}_{S}^{a}$ consists of mapping classes fixing the puncture $a$. Let $V_{\tilde{\omega}}(\hat{z})$ denote the stabilizer of $\Delta_{\tilde{\omega}}(\hat{z})$ in $\operatorname{Mod}_{S}^{a}$. Then $V_{\tilde{\omega}}(\hat{z})$ is a subgroup of the Veech group of $\Delta_{\tilde{\omega}}(\hat{z})$ of finite index. In particular, if $\tilde{S}$ is compact, then $V_{\tilde{\omega}}(\hat{z})$ is the Veech group of $\Delta_{\tilde{\omega}}(\hat{z})$. See Veech [17] and Hubert-Lanneau [8] for discussions on Veech groups of general Teichmüller disks in a Teichmüller space. It is well known (Earle and Gardiner [7]) that there exist Teichmüller disks in $T(S)$ whose Veech groups are trivial.

The main purpose of this article is to prove the following result.

THEOREM 1.1. Let $\tilde{S}$ be a Riemann surface of finite analytic type $(p, n)$ with the genus $p \geq 2$. Then,

(1) For each hyperbolic mapping class $\tilde{\theta}$ of $M o d_{\tilde{S}}$, there exists an uncountable subset $\Omega_{0} \subset \mathbf{H}$ with a full measure such that for each point $\hat{z}_{0} \in \Omega_{0}$, the Teichmüller disk $\Delta_{\tilde{\omega}}(\hat{z}) \subset T(S)$ defined as $(1.6)$ satisfies $j\left(\Delta_{\tilde{\omega}}(\hat{z})\right)=\tilde{\Delta}_{\tilde{\omega}}$ and the stabilizer $V_{\tilde{\omega}}(\hat{z})$ of $\Delta_{\tilde{\omega}}(\hat{z})$ in $\mathrm{Mod}_{S}^{a}$ is trivial. 
(2) There exist hyperbolic mapping classes $\tilde{\theta}$ in $\operatorname{Mod}_{\tilde{S}}$ such that for every point $\hat{z} \in \mathbf{H}$, the group $V_{\tilde{\omega}}(\hat{z})$ either is trivial or contains a purely hyperbolic subgroup of finite index.

This article is organized as follows. In Section 2, we review the Teichmüller existence and uniqueness theorem. In Section 3, we investigate the mapping class $\operatorname{group}_{\operatorname{Mod}_{\tilde{S}}}$ as well as its extension to act on the corresponding Bers fibre space $F(\tilde{S})$. Properties of invariant disks by some mapping classes are also discussed in the section. Section 4 is devoted to the proof of Theorem 1.1. Section 5 includes some remarks.

2. Fixed point sets of special quasi-conformal self-maps of Riemann surfaces. Let $\tilde{S}$ be defined as before, and let $\tilde{S}_{0}$ be a Riemann surface of the same type $(p, n)$. Then $\tilde{S}$ and $\tilde{S}_{0}$ are diffeomorphic. Let $f: \tilde{S} \rightarrow \tilde{S}_{0}$ be a quasi-conformal map. The Teichmüller existence and uniqueness theorem states that in the homotopy class of $f$ there is either a unique conformal map or a unique quasi-conformal map $f_{0}$, called an extremal quasi-conformal map, such that the Beltrami coefficient

$$
\frac{\partial_{\bar{z}} f_{0}}{\partial_{z} f_{0}}=k \frac{\bar{\phi}}{|\phi|} \text { for some real number } k \text { with } 0<k<1,
$$

where $\phi$ is a quadratic differential on $\tilde{S}$ that satisfies (1.3) and may have simple poles at some punctures of $\tilde{S}$. Associated to $f_{0}$ there is another quadratic differential $\psi$ on $\tilde{S}_{0}$. The quadratic differentials $\phi$ and $\psi$ have the same number of zeros and some zeros of $\phi$ and $\psi$ may be punctures of $\tilde{S}$ and $\tilde{S}_{0}$, respectively. If $z$ is a zero of $\phi$, then $f_{0}(z)$ is a zero of $\psi$ of the same order. The quadratic differential $\phi$ (resp. $\psi$ ) determines a pair $\left(\Phi_{h}, \Phi_{v}\right)$ (resp. $\left.\left(\Psi_{h}, \Psi_{v}\right)\right)$ of transverse trajectories on $\tilde{S}$ (resp. $\left.\tilde{S}_{0}\right)$. The map $f_{0}$ sends $\Phi_{h}$ to $\Psi_{h}$ and $\Phi_{v}$ to $\Psi_{v}$ via a stretching map and a compressing map. More precisely, if $P \in \tilde{S}$ is not a zero of $\phi$ and $z$ is a $\phi$-coordinate about $P$, then there is a $\psi$-coordinate $\zeta$ at $f_{0}(z) \in \tilde{S}_{0}$ such that

$$
\zeta \circ f_{0}=\frac{z+k \bar{z}}{\sqrt{1-k^{2}}} .
$$

From (2.1) the map $f_{0}$ can be realized as

$$
\operatorname{Re} \zeta=\left(\frac{1+k}{1-k}\right)^{1 / 2} \operatorname{Re} z \text { and } \operatorname{Im} \zeta=\left(\frac{1+k}{1-k}\right)^{-1 / 2} \operatorname{Im} z
$$

In literature (see e.g. Strebel [13]), $\phi$ and $\psi$ are called the initial and terminal quadratic differentials of $f_{0}$, respectively.

Consider the case $\tilde{S}_{0}=\tilde{S}$. Then $f_{0}$ defines a mapping class of $\operatorname{Mod}_{\tilde{S}}$ and $\phi$ and $\psi$ are differentials on $\tilde{S}$. As an example, for any simple closed geodesic $\tilde{c}$ on $\tilde{S}$, the Dehn twist $t_{\tilde{c}}$ determines a parabolic mapping class of $\operatorname{Mod}_{\tilde{S}}$ (for the definition of parabolic mapping classes, see Bers [6]). According to the Teichmüller theorem, in the homotopy class of $t_{\tilde{c}}$, there is a unique extremal Teichmüller self-map whose corresponding initial and terminal quadratic differentials $\phi$ and $\psi$ are simple Jenkins-Strebel differentials. See Strebel [15] for an exposition.

As another example, for every irreducible self-map $f$ of $\tilde{S}$ (in the sense that it does not keep any curve simplex invariant), we can modify the conformal structure of $\tilde{S}$ so that $f$ is isotopic to an $f_{0}$ that is an absolutely extremal Teichmüller map on a new 
surface $\tilde{S}_{0}$. The choice of $\tilde{S}_{0}$ is not unique and all the surfaces $\tilde{S}_{0}$ so obtained determine a set of points in $T(\tilde{S})$ that constitutes a real Teichmüller geodesic. In this case, we have $\phi=\psi$ and thus $\Phi_{h}=\Psi_{h}$ and $\Phi_{v}=\Psi_{v}$. See Bers [6] and Thurston [16] for more details.

For any quasi-conformal self-map $f$ of $\tilde{S}$, we denote by

$$
\mathscr{S}(f)=\{z \in \tilde{S}: \quad f(z)=z\} .
$$

Let $\tilde{\chi}$ be a non-trivial non-elliptic hyperbolic mapping class of $\tilde{S}$, and let $f_{0}$ be the Teichmüller self-map representing $\tilde{\chi}$. We claim that the set $\mathscr{S}\left(f_{0}\right)$ is finite. In fact more careful examination reveals that $\mathscr{S}\left(f_{0}\right)$ consists of zeros of the corresponding quadratic differential $\phi$. See Lemma 3.1 .

More generally, if $f_{0}: \tilde{S} \rightarrow \tilde{S}$ is a quasi-conformal map whose Beltrami coefficient is $t \mu$ for $t \in \mathbf{D}-\{0\}$ and $\mu=\bar{\phi} /|\phi|$, where $\phi$ is a holomorphic quadratic differential on $\tilde{S}$. We claim that $\mathscr{S}\left(f_{0}\right)$ has measure zero. Suppose that $\mathscr{S}\left(f_{0}\right)$ has measure $>0$. We denote by $z=z(P)$ a local coordinate chart at a point $P \in \tilde{S}$. For each $P \in \mathscr{S}\left(f_{0}\right)$, the function $F(z)=f_{0}(z)-z=0$. This implies that $\partial_{z} F(z)=0$ and $\partial_{\bar{z}} F(z)=0$ for almost all points $z=z(P) \in \mathscr{S}\left(f_{0}\right)$, where the derivatives are taken in the sense of distribution. Since $\partial_{z} F(z)=\partial_{z} f_{0}(z)-1$, we conclude that $\partial_{z} f_{0}(z) \neq 0$ for almost all points $z=z(P) \in \mathscr{S}\left(f_{0}\right)$. On the other hand, $\partial_{\bar{z}} F(z)=\partial_{\bar{z}} f_{0}(z)$. It follows that $\partial_{\bar{z}} f_{0}(z)=0$ for almost all points $z=z(P)$ in $\mathscr{S}\left(f_{0}\right)$. Therefore, the Beltrami coefficient of $f_{0}$ vanishes at almost all points in $\mathscr{S}\left(f_{0}\right)$. But we know that $\mathscr{S}\left(f_{0}\right)$ has measure $>0$ and the Beltrami coefficient of $f_{0}$ is $t \bar{\phi} /|\phi|$ which can not be zero on a set with positive measure (since $\phi$ has isolated zeros). This is a contradiction. We summarize the result in the following lemma.

LEMMA 2.1. Let $\tilde{\chi}$ be a non-trivial non-elliptic mapping class of $\tilde{S}$, and let $f_{0}$ be a quasi-conformal self-map of $\tilde{S}$ representing $\tilde{\chi}$. Assume that $f_{0}$ has a Beltrami coefficient with form $t \bar{\phi} /|\phi|$ for a complex number $t \in \mathbf{D}-\{0\}$ and a holomorphic quadratic differential $\phi$ on $\tilde{S}$. Then the set $\mathscr{S}\left(f_{0}\right)$ defined as in (2.3) has measure zero.

3. Mapping class groups acting on Bers fibre spaces. The mapping class group $\operatorname{Mod}_{\tilde{S}}$ extends to the $\operatorname{group} \bmod (\tilde{S})$ that acts on $F(\tilde{S})$ and preserves the fibre structure of $F(\tilde{S})$. Every element $\theta \in \bmod (\tilde{S})$ can be represented by a self-map $w$ of $\mathbf{H}$. Two such self-maps $\hat{w}_{1}, \quad \hat{w}_{2}$ of $\mathbf{H}$ represent the same element of $\bmod (\tilde{S})$ if $\hat{w}_{1}=\hat{w}_{2}$ on $\partial \mathbf{H}$ and they both project to maps $w_{1}, w_{2}: \tilde{S} \rightarrow \tilde{S}$ isotopic to each other; or equivalently, $\hat{w}_{1} h\left(\hat{w}_{1}\right)^{-1}=\hat{w}_{2} h\left(\hat{w}_{2}\right)^{-1}$ for all elements $h \in G$, write $\theta=\left[\hat{w}_{1}\right]=\left[\hat{w}_{2}\right]$. Then $\theta \in \bmod (\tilde{S})$.

With the aid of the Bers isomorphism $\varphi: F(\tilde{S}) \rightarrow T(S)$, the $\operatorname{group} \bmod (\tilde{S})$ is isomorphic to $\operatorname{Mod}_{S}^{a}$ by a conjugation $\varphi^{*}$ :

$$
\bmod (\tilde{S}) \ni[\hat{w}] \stackrel{\varphi^{*}}{\rightarrow} \varphi \circ[\hat{w}] \circ \varphi^{-1} \in \operatorname{Mod}_{S}^{a} .
$$

It follows that the forgetful map $j: T(S) \rightarrow T(\tilde{S})$ defined as (1.5) also induces a natural projection

$$
i: \operatorname{Mod}_{S}^{a} \rightarrow \operatorname{Mod}_{\tilde{S}}
$$

obtained by forgetting the puncture $a$. For simplicity, we denote throughout the article $[\hat{w}]^{*}=\varphi^{*}([\hat{w}])$. In particular, the group $G$ can be regarded as a normal subgroup of 
$\bmod (\tilde{S})$ and thus it keeps each fibre of $F(\tilde{S})$ invariant. The group $G$ is isomorphic to a subgroup of $\operatorname{Mod}_{S}^{a}$ that consists of all elements $\theta=h^{*}$ with $h \in G$ so that $i\left(h^{*}\right)=$ identity, i.e., $G \cong i^{-1}$ (id). In [11], Kra proved that $h \in G$ is simple hyperbolic if and only if $h^{*}$ is represented by a spin map (that is of the form $t_{\alpha} \circ t_{\beta}^{-1}$, where $t_{\alpha}$ and $t_{\beta}$ are the positive Dehn twists along curves $\alpha$ and $\beta$ that are components of an $a$-punctured cylinder on $S$ ); and is parabolic if and only if $h^{*}$ is represented by a Dehn twist along a curve that bounds a twice punctured disk enclosing $a$.

Now let $\tilde{\theta}, \tilde{\omega}$ and $\phi_{\tilde{\omega}}$ be as introduced in the previous section.

LEMMA 3.1. If $\tilde{\omega}\left(z_{0}\right)=z_{0}$ for some $z_{0} \in \tilde{S}$, then $z_{0}$ is a zero of $\phi_{\tilde{\omega}}$. Further, $\tilde{\omega}$ leaves invariant the set of non-puncture zeros of $\phi_{\tilde{\omega}}$.

Proof. The lemma follows directly from the definition of absolutely extremal Teichmüller self-map. See also (2.1) or (2.2).

Assume that there exists a hyperbolic $\theta \in \operatorname{Mod}_{S}^{a}$ such that $i(\theta)=\tilde{\theta}$. By Bers [6], there exists a unique Teichmüller disk in $T(S)$, denoted by $\Delta_{\theta}$, that is invariant under $\theta$.

LemMA 3.2. With the above conditions, $j\left(\Delta_{\theta}\right) \subset T(\tilde{S})$ is an invariant disk (which may not be a Teichmüller disk) under the action of $\tilde{\theta}$.

Proof. For any point $x \in T(S)$, we have

$$
j(\theta(x))=\tilde{\theta}(j(x))
$$

Now let $y \in j\left(\Delta_{\theta}\right)$, and let $x \in \Delta_{\theta}$ be such that $j(x)=y$. Since $\theta$ is hyperbolic and $x \in \Delta_{\theta}, \theta(x) \in \Delta_{\theta}$. Thus $j(\theta(x)) \in j\left(\Delta_{\theta}\right)$. It follows from (3.2) that $\tilde{\theta}(y)=\tilde{\theta}(j(x))=$ $j(\theta(x)) \in j\left(\Delta_{\theta}\right)$. Since $y \in j\left(\Delta_{\theta}\right)$ is arbitrary, $\tilde{\theta}$ keeps $j\left(\Delta_{\theta}\right)$ invariant.

The following lemma was proved in Kra [11].

Lemma 3.3. Assume that $\phi_{\tilde{\omega}}$ has a non-puncture zero $z_{0}$ so that $\tilde{\omega}\left(z_{0}\right)=z_{0}$. Then there are infinitely many (countable) hyperbolic elements $\theta \in \operatorname{Mod}_{S}^{a}$ such that $i(\theta)=\tilde{\theta}$ and the invariant disk $\Delta_{\theta}$ of $\theta$ is of form $\Delta_{\tilde{\omega}}(\hat{z})$ for some $\hat{z} \in \mathbf{H}$.

From Lemma 3.2 and Lemma 3.3, we obtain the following lemma.

Lemma 3.4. Assume that $\phi_{\tilde{\omega}}$ has a non-puncture zero $z_{0}$ with $\tilde{\omega}\left(z_{0}\right)=z_{0}$. There exist infinitely many (countable) hyperbolic elements $\theta \in \operatorname{Mod}_{S}^{a}$ such that $i(\theta)=\tilde{\theta}$ and the projection (1.5) realizes an isometric embedding of $\Delta_{\theta}$ into $T(\tilde{S})$ with the property that $j\left(\Delta_{\theta}\right)$ is the invariant Teichmüller disk of $\tilde{\theta}$.

REMARK 3.1. It is not clear if there is a hyperbolic element $\theta \in \operatorname{Mod}_{S}^{a}$ with $i(\theta)=\tilde{\theta}$ being hyperbolic such that $\Delta_{\theta}$ is not of the form $\Delta_{\tilde{\omega}}(\hat{z})$, but $j: T(S) \rightarrow T(\tilde{S})$ still induces an isometric embedding of $\Delta_{\theta}$ into $T(\tilde{S})$.

LemMA 3.5. Let $\hat{z}_{1}, \hat{z}_{2} \in \mathbf{H}$ be two points with $\hat{z}_{1} \neq \hat{z}_{2}$. Let $\mathscr{D}_{\tilde{\omega}}\left(\hat{z}_{1}\right), \mathscr{D}_{\tilde{\omega}}\left(\hat{z}_{2}\right)$ be defined as in (1.4). Then $\mathscr{D}_{\tilde{\omega}}\left(\hat{z}_{1}\right)$ is disjoint from $\mathscr{D}_{\tilde{\omega}}\left(\hat{z}_{2}\right)$. Further, every such $\mathscr{D}_{\tilde{\omega}}(\hat{z})$ intersects $\mathbf{H}$ exactly one point.

Proof. To see that $\mathscr{D}_{\tilde{\omega}}\left(\hat{z}_{1}\right)$ is disjoint from $\mathscr{D}_{\tilde{\omega}}\left(\hat{z}_{2}\right)$, we notice that

$$
\mathscr{D}_{\tilde{\omega}}\left(\hat{z}_{i}\right)=\left\{\left([t \mu], w^{t \mu}\left(\hat{z}_{i}\right)\right): t \in \mathbf{D}\right\} .
$$


Suppose that

$$
\left(\left[t_{1} \mu\right], w^{t_{1} \mu}\left(\hat{z}_{1}\right)\right)=\left(\left[t_{2} \mu\right], w^{t_{2} \mu}\left(\hat{z}_{2}\right)\right), \text { for some } t_{1}, t_{2} \in \mathbf{D} .
$$

From Lemma 3.4, we see that

$$
\pi\left(\mathscr{D}_{\tilde{\omega}}\left(\hat{z}_{1}\right)\right)=\pi\left(\mathscr{D}_{\tilde{\omega}}\left(\hat{z}_{2}\right)\right)
$$

is the Teichmüller disk $\Delta_{\tilde{\theta}}$, which means that there is an isometric embedding $\iota: \mathbf{D} \rightarrow$ $\Delta_{\tilde{\theta}}$ that sends $t \in \mathbf{D}$ to $[t \mu]$, from which it follows that $t_{1}=t_{2}=t$. Now from (3.3) we obtain that $w^{t \mu}\left(\hat{z}_{1}\right)=w^{t \mu}\left(\hat{z}_{2}\right)$. Since $\hat{z}_{1} \neq \hat{z}_{2}$ and $w^{t \mu}: \hat{\mathbf{C}} \rightarrow \hat{\mathbf{C}}$ is a quasi-conformal map, $w^{t \mu}\left(\hat{z}_{1}\right) \neq w^{t \mu}\left(\hat{z}_{2}\right)$. This contradicts (3.3). Thus there are no points of $F(\tilde{S})$ lying in both $\mathscr{D}_{\tilde{\omega}}\left(\hat{z}_{1}\right)$ and $\mathscr{D}_{\tilde{\omega}}\left(\hat{z}_{2}\right)$, which says that $\mathscr{D}_{\tilde{\omega}}\left(\hat{z}_{1}\right)$ is disjoint from $\mathscr{D}_{\tilde{\omega}}\left(\hat{z}_{2}\right)$.

Suppose that $\mathscr{D}_{\tilde{\omega}}(\hat{z})$ intersects $\mathbf{H}$ at least twice for some $\hat{z} \in \mathbf{H}$, which implies that the projection $\pi: F(\tilde{S}) \rightarrow T(\tilde{S})$ does not define an embedding of $\mathscr{D}_{\tilde{\omega}}(\hat{z})$ into $T(\tilde{S})$. Thus $\pi\left(\mathscr{D}_{\tilde{\omega}}(\hat{z})\right)$ is not the Teichmüller disk invariant under $\tilde{\theta}$. This contradicts Lemma 3.4.

Let $\chi \in \operatorname{Mod}_{S}^{a}$ be a non-trivial mapping class such that

$$
\chi\left(\Delta_{\tilde{\omega}}(\hat{z})\right)=\Delta_{\tilde{\omega}}(\hat{z})
$$

for a point $\hat{z} \in \mathbf{H}$. Assume that $\tilde{\chi}=i(\chi) \in \operatorname{Mod}_{\tilde{S}}$ is non-trivial and non-elliptic. Let $[\hat{f}] \in \bmod (\tilde{S})$ be the element corresponding to $\chi$. Then we have

$$
[\hat{f}]\left(\mathscr{D}_{\tilde{\omega}}(\hat{z})\right)=\mathscr{D}_{\tilde{\omega}}(\hat{z}) .
$$

By definition (1.4) and Lemma 3.5. $\mathscr{D}_{\tilde{\omega}}(\hat{z}) \cap \mathbf{H}=\hat{z}$.

Caution. We may choose a representative (also denoted by $\hat{f})$ of $[\hat{f}]$ so that $\hat{f}(\hat{z}) \neq \hat{z}$. That is to say, there are quasi-conformal maps $\hat{f}$ such that $\hat{f}(\hat{z}) \neq \hat{z}$ and (3.5) still holds. On the other hand, for quasi-conformal maps $\hat{f}$ with $\hat{f}(\hat{z})=\hat{z}$, there is no guarantee that (3.5) is satisfied.

Despite the complexity mentioned above, when $\hat{f}$ is a quasi-conformal self-map of $\mathbf{H}$ whose Beltrami coefficient is $t \mu$ for $t \in \mathbf{D}-\{0\}$ and $\mu=\overline{\phi_{\tilde{\omega}}} /\left|\phi_{\tilde{\omega}}\right|,(3.5)$ is equivalent to the condition $\hat{f}(\hat{z})=\hat{z}$. More precisely, we establish the following result.

LEMMA 3.6. With the above notation, assume that (3.4) or (3.5) holds. The mapping classes $\chi$ and $\tilde{\chi}$ are represented by quasi-conformal maps $\hat{f}_{0}$ and $f_{0}$, respectively, so that $\hat{f}_{0}(\hat{z})=\hat{z}, f_{0}(z)=z$ for $z=\varrho\left(\mathscr{D}_{\tilde{\omega}}(\hat{z}) \cap \mathbf{H}\right)$ and both have the Beltrami coefficient $t \mu$ for $a$ $t \in \mathbf{D}-\{0\}$ and $\mu=\overline{\phi_{\tilde{\omega}}} /\left|\phi_{\tilde{\omega}}\right|$. Conversely, if $\chi$ is represented by $\hat{f}_{0}$ that has the Beltrami coefficient t $\mu$ and satisfies $\hat{f}_{0}(\hat{z})=\hat{z}$, then (3.4) or (3.5) holds. In particular, if $\tilde{\chi}=\tilde{\theta}$, then $z=z_{0}$ is a non-puncture zero of $\phi_{\tilde{\omega}}$ so that $\tilde{\omega}\left(z_{0}\right)=z_{0}$.

Proof. Since $\chi$ leaves $\Delta_{\tilde{\omega}}(\hat{z})$ invariant, Lemma 3.2 says that $\tilde{\chi}=i(\chi)$ leaves $j\left(\Delta_{\tilde{\omega}}(\hat{z})\right)$ invariant. From Lemma 3.4, $\tilde{\Delta}_{\tilde{\omega}}=j\left(\Delta_{\tilde{\omega}}(\hat{z})\right)$ is the Teichmüller disk determined by the quadratic differential $\phi_{\tilde{\omega}}$. So $\tilde{\chi}$ leaves the Teichmüller disk $\tilde{\Delta}_{\tilde{\omega}}$ invariant.

Since $\tilde{\chi}([0]) \in \tilde{\Delta}_{\tilde{\omega}}$, we can write $\tilde{\chi}([0])=\left[t_{1} \mu\right]$ for a complex number $t_{1} \in \mathbf{D}-\{0\}$, where $\mu=\overline{\phi_{\tilde{\omega}}} /\left|\phi_{\tilde{\omega}}\right|$. There is a quasi-conformal self-map $f_{0}$ of $\tilde{S}$ such that $f_{0}$ represents $\tilde{\chi}$ and has Beltrami coefficient $t_{1} \mu$. An easy computation shows that $f_{0}^{-1}$ has Beltrami coefficient $t_{2} \mu$, where $t_{2}$ is a complex number with $\left|t_{2}\right|<1$ and $\left|t_{1} / t_{2}\right|=1$. 
Let $\hat{f}_{0}: \mathbf{H} \rightarrow \mathbf{H}$ denote the lift of $f_{0}$ satisfying $\left[\hat{f}_{0}\right]^{*}=\chi$. Then we know that

$$
\tilde{\chi}([0])=\left[\text { Beltrami coefficient of } \hat{f}_{0}^{-1}\right] \in \tilde{\Delta}_{\tilde{\omega}} .
$$

Now (3.4) tells us that $\chi \circ \varphi\left(\mathscr{D}_{\tilde{\omega}}(\hat{z})\right)=\varphi\left(\mathscr{D}_{\tilde{\omega}}(\hat{z})\right)$. Thus

$$
\left[\hat{f}_{0}\right]\left(\mathscr{D}_{\tilde{\omega}}(\hat{z})\right)=\mathscr{D}_{\tilde{\omega}}(\hat{z}) .
$$

From direct computations similar to the proof of Theorem 1 of [11], we know that the Beltrami coefficient of $w^{t \mu} \circ \hat{f}_{0}^{-1}$ for any complex number $t \in \mathbf{D}$ is $\xi(t) \mu$, where $\xi(t)$ is a complex number defined as

$$
\xi(t)=-\frac{t_{2}}{t_{1}} \frac{t-t_{1}}{1-t \bar{t}_{1}} .
$$

Hence $\xi: \mathbf{D} \rightarrow \mathbf{D}$ is a conformal automorphism.

Notice that $\hat{z}=\mathscr{D}_{\tilde{\omega}}(\hat{z}) \cap \mathbf{H}$. It then follows from the argument of Proposition 3 of [11] that the action of $\left[\hat{f}_{0}\right]$ can be written as

$$
\left[\hat{f}_{0}\right]\left([t \mu], w^{t \mu}(\hat{z})\right)=\left([\xi(t) \mu], w^{\xi(t) \mu} \circ \hat{f}_{0}(\hat{z})\right) .
$$

As $t$ runs over $\mathbf{D}, \xi(t)$ runs over $\mathbf{D}$ as well. This says that

$$
\left[\hat{f}_{0}\right]\left(\mathscr{D}_{\tilde{\omega}}(\hat{z})\right)
$$

is a disk of the form (1.4) and passes through the point $\hat{f}_{0}(\hat{z}) \in \mathbf{H}$.

Denote by $\mathscr{D}_{0}=\left[\hat{f}_{0}\right]\left(\mathscr{D}_{\tilde{\omega}}(\hat{z})\right)$. Assume that $\hat{f}_{0}(\hat{z}) \neq \hat{z}$. By Lemma $3.5, \mathscr{D}_{0} \neq \mathscr{D}_{\tilde{\omega}}(\hat{z})$. Hence $\varphi\left(\mathscr{D}_{0}\right) \neq \varphi\left(\mathscr{D}_{\tilde{\omega}}(\hat{z})\right)=\Delta_{\tilde{\omega}}(\hat{z})$. But

$$
\varphi\left(\mathscr{D}_{0}\right)=\varphi \circ\left[\hat{f}_{0}\right]\left(\mathscr{D}_{\tilde{\omega}}(\hat{z})\right)=\left[\hat{f}_{0}\right]^{*} \circ \varphi\left(\mathscr{D}_{\tilde{\omega}}(\hat{z})\right)=\left[\hat{f}_{0}\right]^{*}\left(\Delta_{\tilde{\omega}}(\hat{z})\right) .
$$

From (3.7), we have $\left[\hat{f}_{0}\right]^{*}\left(\Delta_{\tilde{\omega}}(\hat{z})\right)=\Delta_{\tilde{\omega}}(\hat{z})$. This leads to a contradiction, proving that $\hat{f}_{0}(\hat{z})=\hat{z}$. Set $z=\varrho(\hat{z})$. From $\varrho \circ \hat{f}_{0}=f_{0} \circ \varrho$ we see that $f_{0}(z)=z$.

The converse part of the result is included in the argument of Theorem 1 of [11]. Finally, if $f_{0}=\tilde{\omega}$, then $\tilde{\omega}(z)=z$. In particular, $z$ cannot be a puncture of $\tilde{S}$. Since $\tilde{\omega}$ is an absolutely extremal Teichmüller map on $\tilde{S}$, by Lemma $3.1, z=z_{0}$ is a non-puncture zero of the quadratic differential $\phi_{\tilde{\omega}}$.

From Lemma 3.3, we know that if there is a point $z_{0} \in \tilde{S}$ that is a non-puncture zero of $\phi_{\tilde{\omega}}$ with $\tilde{\omega}\left(z_{0}\right)=z_{0}$, then for any point $\hat{z}_{0} \in \mathbf{H}$ with $\varrho\left(\hat{z}_{0}\right)=z_{0}$, the stabilizer $V_{\tilde{\omega}}\left(\hat{z}_{0}\right)$ of $\Delta_{\tilde{\omega}}\left(\hat{z}_{0}\right)$ in the pointed mapping class group $\operatorname{Mod}_{S}^{a}$ contains a hyperbolic mapping class $\theta$ with $i(\theta)=\tilde{\theta}$. This particularly implies that $V_{\tilde{\omega}}\left(\hat{z}_{0}\right)$ is not empty and contains at least an infinite cyclic subgroup generated by $\theta$.

4. Proof of Theorem 1.1. By Hurwitz's theorem (see e.g. Theorem V.1.3 of Farkas and Kra [9]), the group of conformal automorphisms of $\tilde{S}$ is finite, and the order of the group is $<84(p-1)$. For each conformal automorphism $\zeta$ of $\tilde{S}$, the quotient $\tilde{S} /\langle\zeta\rangle$ is an orbifold of finite type. So by a theorem of Kravetz [12], the number of fixed points of $\zeta$ on $\tilde{S}$ is finite. Let $A$ be the set of points $z$ of $\tilde{S}$ such that there is a non-trivial conformal automorphism $\zeta$ with $\zeta(z)=z$. We conclude that $A$ is finite. 
It is well known (see Abikoff [1]) that the mapping class group $\operatorname{Mod}_{\tilde{S}}$ is discrete when viewed as a group of holomorphic automorphisms of $T(\tilde{S})$. By Lemma 2.1, for each non-conformal quasi-conformal self-map $f$ of $\tilde{S}$, whose Beltrami coefficient is $\overline{t \phi_{\tilde{\omega}}} /\left|\phi_{\tilde{\omega}}\right|$, the set

$$
B_{f}=\{P \in \tilde{S}: f(P)=P\}
$$

has measure zero. We conclude that the union

$$
B=\bigcup B_{f}
$$

for all such maps $f$ has measure zero. By combining $A$ and $B$, we see that $A \cup B$ has measure zero. Hence the set

$$
\Sigma=\{\hat{z} \in \mathbf{H}: \varrho(\hat{z}) \in A \cup B\}
$$

has measure zero. Denote by

$$
\Omega=\mathbf{H}-\Sigma,
$$

where $\Sigma$ is defined as (4.1). Then $\Omega$ is uncountable with a full measure. We prove the following lemma.

LeMma 4.1. For any $\hat{z} \in \Omega$, the group $V_{\tilde{\omega}}(\hat{z})$ is either trivial or finitely cyclic.

Proof. Suppose that $V_{\tilde{\omega}}(\hat{z})$ contains an element $\chi \in \operatorname{Mod}_{S}^{a}$ with infinite order. That is,

$$
\chi\left(\Delta_{\tilde{\omega}}(\hat{z})\right)=\Delta_{\tilde{\omega}}(\hat{z}) \text { and } \chi^{n} \neq \text { id for any integer } n .
$$

Since $\chi$ is not elliptic, from the Bers classification for mapping classes [6], $\chi$ is either parabolic, hyperbolic, or pseudo-hyperbolic.

From Theorem 1 of Kra [11], any pseudo-hyperbolic mapping class $\chi$ cannot keep $\Delta_{\tilde{\omega}}(\hat{z})$ invariant. To see this fact, we note that for a Teichmüller disk $\Delta_{\tilde{\omega}}(\hat{z})$, there is an isometry

$$
\iota: \mathbf{D} \rightarrow \Delta_{\tilde{\omega}}(\hat{z})
$$

with respect to the hyperbolic metric on $\mathbf{D}$ and the Teichmüller metric on $\Delta_{\tilde{\omega}}(\hat{z})$. If there exists a pseudo-hyperbolic mapping class $\chi$ keeping $\Delta_{\tilde{\omega}}(\hat{z})$ invariant, then $\iota^{-1} \circ \chi \circ \iota \in \operatorname{Aut}(\mathbf{D})$ is a non-trivial Möbius transformation that is neither elliptic, nor parabolic, nor hyperbolic. This is absurd.

Via the isometry (4.2), $\chi$ determines a Möbius transformation $A_{\chi}$ on D. So $A_{\chi}$ is either trivial or non-trivial. If $A_{\chi}$ is trivial, then $\Delta_{\tilde{\omega}}(\hat{z})$ is contained in a fixed-point locus of $\chi$. In this case, if we write $[\hat{f}]^{*}=\chi$, then $[\hat{f}] \in \bmod (\tilde{S})$ determines a conformal automorphism $\zeta$ of $\tilde{S}$. Since $\Delta_{\tilde{\omega}}(\hat{z}) \cap \mathbf{H}=\hat{z}, \zeta$ fixes the point $z=\varrho(\hat{z}) \in \tilde{S}$. It follows by definition that $z \in A$. Hence $\hat{z} \in \Sigma$. But this contradicts that $\hat{z} \in \Omega$. If $A_{\chi}$ is non-trivial, there are three cases to consider: $A_{\chi}$ is either hyperbolic, parabolic, or elliptic.

Case 1. $A_{\chi}$ is hyperbolic. Then $A_{\chi}$ has an invariant geodesic $l \subset \mathbf{D}$. Hence $\chi$ has an invariant Teichmüller geodesic $\iota(l)$. From Theorem 6 of Bers [6], $\chi$ is hyperbolic.

If $\tilde{\chi}=i(\chi) \in \operatorname{Mod}_{\tilde{S}}$ is non-trivial and non-elliptic, then we notice that $\mathscr{D}_{\tilde{\omega}}(\hat{z}) \cap$ $\mathbf{H}=\hat{z}$. From Lemma 3.6, we assert that $\tilde{\chi}$ is represented by a quasi-conformal map 
$f_{0}$ that has a Beltrami coefficient $t \mu$ for some $t \in \mathbf{D}-\{0\}$ and satisfies $f_{0}(z)=z$. This means $z \in B$. Thus $\hat{z} \in \Sigma$ and $\hat{z} \notin \Omega$. This is a contradiction.

If $\tilde{\chi}$ is trivial, then there is an element $g \in G$ such that $g^{*}=\chi$ (in fact, by Theorem 2 of [11], $g$ is an essential hyperbolic element). Now $g$ acts on $\mathbf{H}$ without any fixed points, so from Lemma $3.5, g\left(\mathscr{D}_{\tilde{\omega}}(\hat{z})\right)$ is disjoint from $\mathscr{D}_{\tilde{\omega}}(\hat{z})$, which in turn implies that $g^{*}\left(\Delta_{\tilde{\omega}}(\hat{z})\right)$ is disjoint from $\Delta_{\tilde{\omega}}(\hat{z})$. But $g^{*}=\chi$. It turns out that $\chi\left(\Delta_{\tilde{\omega}}(\hat{z})\right)$ is disjoint from $\Delta_{\tilde{\omega}}(\hat{z})$, contradicting that $\chi\left(\Delta_{\tilde{\omega}}(\hat{z})\right)=\Delta_{\tilde{\omega}}(\hat{z})$.

If $\tilde{\chi}$ is elliptic, then there is an integer $k \geq 2$ such that $\tilde{\chi}^{k}(\tilde{x})=\tilde{x}$ for a point $\tilde{x} \in T(\tilde{S})$. Let $x \in \mathscr{D}_{\tilde{\omega}}(\hat{z})$ be such that $\pi(x)=\tilde{x}$. Since $\chi$ leaves invariant $\Delta_{\tilde{\omega}}(\hat{z})$, and since $i(\chi)=\tilde{\chi}$, we obtain

$$
\pi \circ \chi^{k}(x)=\tilde{\chi}^{k}(\tilde{x})=\tilde{x}=\pi(x) .
$$

But we know that the restriction of $\pi$ to $\mathscr{D}_{\tilde{\omega}}(\hat{z})$ is an embedding. It follows from (4.3) that $\chi^{k}(x)=x$. This implies that $A_{\chi}$ is a non-trivial elliptic Möbius transformation. So Case 1 can not occur, and we conclude that $\chi$ is not hyperbolic.

Case 2. $A_{\chi}$ is parabolic. By taking a suitable power if necessary, we may assume that $\chi$ is represented by multi twists along a system

$$
\left\{c_{1}, \ldots, c_{s}\right\}, \quad s \geq 1
$$

of disjoint simple closed geodesics on $S$. If $s \geq 2$, or $s=1$ but $c_{1}$ projects a non-trivial geodesic on $\tilde{S}$, then $\tilde{\chi}=i(\chi)$ is non-trivial. We can use Lemma 3.6 to conclude that $\tilde{\chi}$ can be represented by a quasi-conformal map $f_{0}$ that has a Beltrami coefficient $t \mu$ for some $t \in \mathbf{D}-\{0\}$ and satisfies $f_{0}(z)=z$ for $z=\varrho(\hat{z})$. This means $z \in B$ and so $\hat{z} \in \Sigma$; that is, $\hat{z} \notin \Omega$. Once again, this is a contradiction.

If $s=1$ and $c_{1}$ projects to the trivial mapping class of $\tilde{S}$, then in this case, $c_{1}$ bounds a twice punctured disk on $S$ that encloses $a$ and another puncture of $\tilde{S}$. This implies that there is a parabolic element $g \in G$ with $g^{*}=\chi$. By the same argument as in Case 1 , we see that $\chi\left(\Delta_{\tilde{\omega}}(\hat{z})\right)$ is disjoint from $\Delta_{\tilde{\omega}}(\hat{z})$, contradicting the fact that $\chi\left(\Delta_{\tilde{\omega}}(\hat{z})\right)=\Delta_{\tilde{\omega}}(\hat{z})$. Hence $\chi$ cannot be parabolic.

Case 3. $A_{\chi}$ is elliptic. We can conclude the proof of Lemma 4.1 by proving the following result.

LEMMA 4.2. The group $V_{\tilde{\omega}}(\hat{z}), \hat{z} \in \Omega$, only contains elliptic elements with a common fixed point in $\Delta_{\tilde{\omega}}(\hat{z})$.

Proof. If $V_{\tilde{\omega}}(\hat{z})$ contains another elliptic element $\chi^{\prime}$ with distinct fixed points, then via (4.2), we obtain two elliptic Möbius transformations $M$ and $M^{\prime}$ with distinct fixed points. According to Theorem 7.39.2 of Beardon [4], the commutator $\left[M, M^{\prime}\right]$ is hyperbolic. Hence $\iota \circ\left[M, M^{\prime}\right] \circ \iota^{-1} \in V_{\tilde{\omega}}(\hat{z})$ is hyperbolic and by the above argument, this is impossible. Therefore, $V_{\tilde{\omega}}(\hat{z})$ is at most finitely cyclic.

This also completes the proof of Lemma 4.1 .

Denote by

$$
\mathscr{F}=\pi^{-1}\left(\tilde{\Delta}_{\tilde{\omega}}\right)=\left\{x \in F(\tilde{S}): \pi(x) \in \tilde{\Delta}_{\tilde{\omega}}\right\} .
$$

To complete the proof of Theorem 1.1 (1), we need to identify those points $\hat{z}_{n} \in \Omega$ for which $\Delta_{\tilde{\omega}}\left(\hat{z}_{n}\right)$ may be invariant under some elliptic mapping classes in $\operatorname{Mod}_{S}^{a}$. We then remove those $\hat{z}_{n}$ from $\Omega$. To accomplish this goal, we first prove the following lemma. 
LemmA 4.3. Let $\chi \in \operatorname{Mod}_{S}^{a}$ be elliptic and keeps $\Delta_{\tilde{\omega}}(\hat{z})$ invariant. Let $A_{\chi}$ be defined under the isometry (4.2). Assume that $A_{\chi}$ is a non-trivial elliptic element. Let $y_{0} \in \mathbf{D}$ be the fixed point of $A_{\chi}$. Then $v_{0}=\iota\left(y_{0}\right)$ is the only fixed point of $\chi$ in $\varphi(\mathscr{F})$.

Proof. Obviously, $\chi\left(v_{0}\right)=v_{0}$ and $\chi$ fixes no other points of $\Delta_{\tilde{\omega}}(\hat{z})$. Let $x_{0} \in \mathscr{D}_{\tilde{\omega}}(\hat{z})$ be such that $\varphi\left(x_{0}\right)=v_{0}$. Denote by $[v]=\pi\left(x_{0}\right) \in T(\tilde{S})$.

Suppose that $\chi$ also fixes a point $v \in \varphi(\mathscr{F})$ with $v \neq v_{0}$. Write $v=\varphi(x)$ for some $x \in \mathscr{F} \subset F(\tilde{S})$. Since the element $[\hat{f}] \in \bmod (\tilde{S})$, where $[\hat{f}]^{*}=\chi$, is fibre preserving, and since $[\hat{f}]$ keeps $\mathscr{D}_{\tilde{\omega}}(\hat{z})$ invariant, $x$ and $x_{0}$ lie in the same fibre $\pi^{-1}([\nu]) \subset \mathscr{F}$ over $[v]$. From the assumption, the restriction of $[\hat{f}]$ to the fibre $\pi^{-1}([v])$ is conformal and fixes the two points $x$ and $x_{0}$. So the restriction of $[\hat{f}]$ to $\pi^{-1}([v])$ must be the identity. On the other hand, the action of $[\hat{f}]$ on the fibre is written as

$$
[\hat{f}]([v], \hat{z})=\left([v], w^{v} \circ \hat{f} \circ\left(w^{\nu}\right)^{-1}(\hat{z})\right) .
$$

We see that $\hat{z}=w^{v} \circ \hat{f} \circ\left(w^{v}\right)^{-1}(\hat{z})$, or $\hat{f} \circ\left(w^{v}\right)^{-1}(\hat{z})=\left(w^{\nu}\right)^{-1}(\hat{z})$. Since $\left(w^{v}\right)^{-1}(\hat{z}) \in \mathbf{H}$ is an arbitrary point, we conclude that $[\hat{f}]$ is trivial. This is again a contradiction.

Now from Lemma 4.3, we know that there exist at most countably many elliptic mapping classes $\chi_{n}$ each of which, when viewed as an automorphism of $\varphi(\mathscr{F})$, has a single distinct fixed point $v_{n}$ in $\varphi(\mathscr{F})$. Assume that $\chi_{n}$ keeps $\Delta_{\tilde{\omega}}\left(\hat{z}_{n}\right)$ invariant. We claim that the only fixed point $v_{n}$ of $\chi_{n}$ must lie in $\Delta_{\tilde{\omega}}\left(\hat{z}_{n}\right)$.

Indeed, by $\iota_{n}: \mathbf{D} \rightarrow \Delta_{\tilde{\omega}}\left(\hat{z}_{n}\right)$ we denote the corresponding isometry. Then $\chi_{n}$ defines a trivial or elliptic Möbius transformation $A_{\chi_{n}}$. If $A_{\chi_{n}}$ is trivial, we use the previous argument to show that this is impossible. If $A_{\chi_{n}}$ is non-trivial, $A_{\chi_{n}}$ has a unique fixed point $y_{n}$ in $\mathbf{D}$. Thus $\iota_{n}\left(y_{n}\right)=v_{n}$ is fixed by $\chi_{n}$ and $v_{n} \in \Delta_{\tilde{\omega}}\left(\hat{z}_{n}\right)$.

Moreover, from Lemma 4.2 we conclude that $\hat{z}_{n} \neq \hat{z}_{m}$ whenever $m \neq n$. Hence by Lemma 3.5, $\Delta_{\tilde{\omega}}\left(\hat{z}_{n}\right)$ is disjoint from $\Delta_{\tilde{\omega}}\left(\hat{z}_{m}\right)$ whenever $m \neq n$. It follows that there exist at most countably many points $\hat{z}_{n} \in \Omega$ such that $\Delta_{\tilde{\omega}}\left(\hat{z}_{n}\right)$ contains $v_{n}$ and does not contain any other $v_{m}$. Therefore, if we set

$$
\Omega_{0}=\Omega-\bigcup_{n \geq 0}\left\{z_{n}\right\},
$$

then $\Omega_{0}$ is an uncountable subset of $\mathbf{H}$ with a full measure and for any $\hat{z} \in \Omega_{0}, \Delta_{\tilde{\omega}}(\hat{z})$ avoids all single fixed points of elliptic mapping classes of $\operatorname{Mod}_{S}^{a}$. Hence $\Delta_{\tilde{\omega}}(\hat{z}), \hat{z} \in \Omega_{0}$, can not be an invariant disk by any elliptic mapping class of $\operatorname{Mod}_{S}^{a}$. This proves (1) of Theorem 1.1.

(2) For a polynomial

$$
P(x)=x^{n}-x^{n-1}-\cdots-x-1
$$

with degree $n \geq 3$, there is a real $\operatorname{root} c>1$ and all other roots are within the unit circle. In literature $c$ is called the Pisot root. The main result of Arnoux and Yoccoz [3] states that there exists a non-conformal absolutely extremal Teichmüller self-mapping $\tilde{\omega}$ on a surface whose maximal dilatation $K(\tilde{\omega})$ satisfies the condition that $K(\tilde{\omega})^{1 / 2}=c$.

Let $\tilde{\theta}$ be the hyperbolic mapping class represented by $\tilde{\omega}$. Let $\tilde{\Delta}_{\tilde{\omega}}$ be the Teichmüller disk in $T(\tilde{S})$ invariant under the action of $\tilde{\theta}$. We see that for any $\hat{z} \in \mathbf{H}, \Delta_{\tilde{\omega}}(\hat{z})$ defined as (1.6) is a Teichmüller disk in $T(S)$. For any $\hat{z} \in \Omega_{0}$, from (1) of Theorem $1.1, V_{\tilde{\omega}}(\hat{z})$ is trivial. So it remains to consider those points $\hat{z}$ in $\mathbf{H}-\Omega_{0}$. We claim that $V_{\tilde{\omega}}(\hat{z})$ for $\hat{z} \in \mathbf{H}-\Omega_{0}$ does not contain any parabolic mapping classes. 
Suppose that there is a parabolic mapping class $\chi \in \operatorname{Mod}_{S}^{a}$ such that $\chi\left(\Delta_{\tilde{\omega}}(\hat{z})\right)=$ $\Delta_{\tilde{\omega}}(\hat{z})$ for $\hat{z} \in \mathbf{H}-\Omega_{0}$. By taking a suitable power if necessary, we assume that $\chi$ is reduced by the curve system (4.4). If $s=1$ and $c_{1}$ bounds a twice punctured disk $D$, then $\chi$ is represented by the Dehn twist $t_{\partial D}$. So $i(\chi)$ is trivial. By Theorem 2 of [11], there is a parabolic element $g \in G$ with $g^{*}=\chi$. Then we can use the same argument of Lemma 4.1 to conclude that this cannot happen. If $s>1$ or $s=1$ but $c_{1}$ does not bound any twice punctured disk, then $\tilde{\chi}=i(\chi)$ is a non-trivial parabolic mapping class. From Lemma 3.4, we have $j\left(\Delta_{\tilde{\omega}}(\hat{z})\right)=\tilde{\Delta}_{\tilde{\omega}}$. By Lemma 3.2, $\tilde{\chi}$ leaves invariant the disk $\tilde{\Delta}_{\tilde{\omega}}$. It follows that the Veech group of $\tilde{\Delta}_{\tilde{\omega}}$ contains a parabolic element $\tilde{\chi}$. This contradicts Corollary 1.3 of Hubert and Lanneau [8].

We conclude that for any point $\hat{z} \in \mathbf{H}$, the group $V_{\tilde{\omega}}(\hat{z})$ is either trivial or only contains hyperbolic and elliptic elements. Now the argument of Corollary 1.5 of [8] can be carried over to our case: any elliptic mapping class is induced by a conformal automorphism $\zeta$ of some surface. So by Hurwitz's theorem (see Theorem V.1.3 of [9]), the order of $\zeta$ is bounded above by $84(p-1)$. According to Theorem 7 of Purzitsky [14], We conclude that for any point $\hat{z} \in \mathbf{H}$, the group $V_{\tilde{\omega}}(\hat{z})$, if non-trivial, must contain a finite index subgroup consists of only hyperbolic elements. This proves (2) of Theorem 1.1.

5. Some remarks. (1) We outline the proof of Corollary 1.3 of [8] as follows. Details can be found in [8]. The polynomial (4.5) of degree $n \geq 3$ has two real roots if $n$ is even and only one real root if $n$ is odd. Since $c$ is the Pisot root, one proves that $\mathbb{Q}[c]$ is not totally real. A computation shows that $\mathbb{Q}\left[c+c^{-1}\right]$ is not totally real either. On the other hand, if $\Delta_{\tilde{\omega}}$ is stabilized by the hyperbolic mapping class $\tilde{\theta}$ that is represented by a product of two parabolic mapping classes, then Theorem 1.1 of [8] asserts that (by using the results of Arnoux and Yoccoz [3] and Kenyon and Smillie [13]) $\mathbb{Q}\left[c+c^{-1}\right]$ can be identified with the trace field which must be totally real, which leads to a contradiction.

(2) The argument of Theorem 1.1 (1) yields that there is an uncountable set $\Omega_{0}$ of $\mathbf{H}$ with a full measure such that the Veech group of each disk $\Delta_{\tilde{\omega}}(\hat{z})$ for $\hat{z} \in \Omega_{0}$ is at most finitely cyclic with order $n+1$. Indeed, by Theorem 10 of Bers [5], the group $\operatorname{Mod}_{S}^{a}$ is a subgroup of $\operatorname{Mod}_{S}$ with index $n+1$. Suppose that there is a non-trivial $\chi \in \operatorname{Mod}_{S}$ such that $\chi\left(\Delta_{\tilde{\omega}}(\hat{z})\right)=\Delta_{\tilde{\omega}}(\hat{z})$. If $\chi$ is hyperbolic or parabolic, then $\chi^{n+1} \in \operatorname{Mod}_{S}^{a}$ is also hyperbolic or parabolic, and the argument remains the same. If $\chi$ is elliptic with $\chi^{n+1}$ being non-trivial, our argument is still valid. It follows that for $\hat{z} \in \Omega_{0}$, the Veech group $V$ of $\Delta_{\tilde{\omega}}(\hat{z})$ only consists of elliptic elements $\chi_{i}$ with $\chi_{i}^{n+1}=\mathrm{id}$. Hence, by the Nielsen realization theorem (see Kerckhoff [10]), $V$ itself is finitely cyclic of order $n+1$.

ACKNOWLEDGMENT. I am indebted to the anonymous referee for pointing out errors and for his valuable comments and suggestions on my previous version of the article.

\section{REFERENCES}

1. W. Abikoff, The real analytic theory of Teichmüller spaces, Lecture notes in mathematics 820 (Springer-Verlag, Berlin-New York, 1980).

2. L. V. Ahlfors and L. Bers, Riemann's mapping theorem for variable metrics, Ann. Math. 72(2) (1960), 385-404. 
3. P. Arnoux and J. C. Yoccoz, Construction de diffeomorphismes pseudo-Anosov, $C$. $R$. Acad. Sci. Paris Sr. I Math. 292(1) (1981), 75-78.

4. A. Beardon, The geometry of discrete groups (Springer-Verlag, New York, 1983).

5. L. Bers, Fiber spaces over Teichmüller spaces, Acta Math. 130 (1973), 89-126.

6. L. Bers, An extremal problem for quasiconformal mappings and a theorem by Thurston, Acta Math. 141 (1978), 73-98.

7. C. J. Earle and F. Gardiner, Teichmüller disks and Veech's $\mathcal{F}$-structures, Contemp. Math. 201 (1997), 165-189.

8. P. Hubert and E. Lanneau, Veech group without parabolic elements, Duke Math. J. 133 (2006), 335-346.

9. H. M. Farkas and I. Kra, Riemann surfaces (Springer-Verlag, New York, 1980).

10. S. P. Kerckhoff, The Nielsen realisation problem, Ann. Math. 117 (1983), 235-265.

11. I. Kra, On the Nielsen-Thurston-Bers type of some self-maps of Riemann surfaces, Acta Math. 146 (1981), 231-270.

12. S. Kravetz, On the geometry of Teichmüller spaces and the structure of their modular groups, Ann. Acad. Sci. Fenn. Math. 278 (1959), 135.

13. R. Kenyon and J. Smillie, Billiards in rational-angled triangles, Comment. Math, Helv. 75 (2000), 65-108.

14. N. Purzitsky, A cutting and pasting of noncompact polygons with applications to Fuchsian groups, Acta Math. 143 (1979), 233-250.

15. K. Strebel, Quadratic differentials (Springer-Verlag, Berlin, 1984).

16. W. P. Thurston, On the geometry and dynamics of diffeomorphisms of surfaces. Bull. Amer. Math. Soc. (N.S.) 19 (1988), 417-431.

17. W. A. Veech, Teichmüller curves in moduli space, Einstein series and an application to triangular billiards. Invent. Math. 97 (1989), 553-583. 